\title{
Potential effects of "social" distancing measures and school lockdown on child and adolescent mental health
}

\author{
Vera Clemens ${ }^{1,2} \cdot$ Peter Deschamps ${ }^{3,4}$. Jörg M. Fegert ${ }^{2,5}$. Dimitris Anagnostopoulos ${ }^{5,6} \cdot$ Sue Bailey ${ }^{3,7}$. \\ Maeve Doyle $e^{5,8}$. Stephan Eliez ${ }^{5,9}$. Anna Sofie Hansen ${ }^{3,10}$. Johannes Hebebrand ${ }^{5,11}$. Manon Hillegers ${ }^{5,12}$. \\ Brian Jacobs $^{3,13}$ - Andreas Karwautz ${ }^{5,14}$ - Eniko Kiss ${ }^{5,15} \cdot$ Konstantinos Kotsis $^{5,16}$ • Hojka Gregoric Kumperscak ${ }^{3,17}$. \\ Milica Pejovic-Milovancevic ${ }^{5,18,19}$. Anne Marie Råberg Christensen ${ }^{5,20}$ • Jean-Philippe Raynaud ${ }^{5,21}$. \\ Hannu Westerinen ${ }^{3,22}$ - Piret Visnapuu-Bernadt ${ }^{3,23}$
}

Published online: 23 May 2020

(c) Springer-Verlag GmbH Germany, part of Springer Nature 2020

So-called "social distancing" and measures of hygiene have proven to be effective reactions to the threat of increasing numbers of COVID-19 cases and fatalities. However, there is no such thing as a free lunch. As medical doctors, we know very well that the majority of our most effective treatment methods unfortunately have chance of provoking severe side effects. In every day practice, we are used to balanced and shared decision-making based on national or international guidelines on an individual level together with our patients and their families and caregivers.

In the battle we are all fighting against COVID 19, at the moment there is no balancing or shared decision-making at an individual level. Rather, at the population level, within their national borders, countries all over the world aim to reduce contact among humans to prevent infection with COVID-19 ${ }^{1}$. This has a positive effect on protecting health, especially for the elderly and ill people. Children, adolescents and their parents are usually at a much lower risk for severe illness, even if there have been rare deaths in this age range as well. Political measures that aim to achieve social distancing hit their age groups particularly although the efficacy of school closures is of equivocal evidence [1]. This calls for further reflection on the effects on their mental health.

Over the past few weeks, an estimated 90\% [2] or more of children and adolescents have faced the effects of school closures. In addition, most other social and out-of-home activities for children and adolescents have been canceled. They no longer enjoy positive interactions with their sport

Vera Clemens, Peter Deschamps and Jörg M. Fegert drafted and organized the paper and contributed equally.

Extended author information available on the last page of the article coaches, music teachers, friends and peers. Children and their families have to share a restricted space at home with limited resources and have to change their daily life and routines to cope with numerous new challenges. Children are supposed to get home schooling, supervised by parents. However, some parents are expected to work as much as possible at home office or, due to the nature of their job, are confronted with daycare problems. Support and the help of grandparents and other family members fall away, as they should avoid contact. All family members struggle with their own anxieties in this situation and for many families, economic pressure further increases stress. When a family member dies, the child has to deal with their grief. Quite often, a combination of challenges clusters in families with limited space, job loss, and other known risk factors such as mental illness/disabilities of family members or single parenting. Together, all of this can erupt in interpersonal violence and there is a series of reports on increased domestic violence and increased child abuse during this first phase of the pandemic (an overview is given by Fegert et al. 2020 [3]). In brief, children and adolescents are at home with their families, experience an increase in stress and a reduction in support and coping resources.

As a result, on the spectrum from healthy-coping-struggling to unwell, many children can expect to suffer, though some will do better. As with most other stressors, resilience and coping are bound to play their role for most individuals. Special attention is needed for those who were already struggling or unwell before home quarantine. Anecdotally, in clinical practice, we see three emerging patterns. A first group of children seem to prosper. They are at home in a quieter environment; they thrive with the structure and support their

\footnotetext{
$\overline{1 \text { COVID-19 }}$ is also known as SARS-CoV-2.
} 
parents provide; they enjoy online learning and they are not exposed to bullying nor find themselves socially excluded. A second group seems to be mildly adversely affected. Their developmental opportunities are paused as they may have too few resources available for online education, are unable to interact with others to practice social skills and no longer have access to practice what they were learning in social/ emotional-treatment. The third group includes children and young people who find themselves in families with increasing negative interactions and who are deprived of the safe haven offered by their schools.

To make matters worse, services and help may be temporarily less available. For those who are in need of professional help, the outreach of mental health services has been reduced. Families avoid consultations due to fear of COVID19. The activity of child protection services and currently existing programs of support or supervision by youth welfare agencies is reduced or interrupted. This is a result of re-organization of services, with provisional care (including re-assigning doctors and nurses not usually involved in critical care), (partial) closures of facilities to avoid the risk of infection and in some services illness among personnel. The lack of access to the support services can be particularly harmful for vulnerable children and/or families who experience increased stress. Thus, school closures exacerbate inequities, disproportionately affecting already disadvantaged children [4].

A small group of youth and children who were previously in residential centers are in a mental health crisis after they went back to their dysfunctional families. In inpatient treatment, infections among staff and/or patients can lead to isolation and separation of patients and sub-groups developing a cohort immunity. A first screening of patients via helplines may help to avoid infections; while in some places, strict rules to avoid infections with distancing and quarantine are abandoned to maintain the functioning of the clinical units. If inpatients have reduced access to their families due to hygiene restrictions, this makes it difficult to adhere to the UN convention of children's rights. Units should maintain their child patient's contact with their family as much as possible.

How are we, as European child and adolescent psychiatrists (CAP), supposed to deal with this situation? First, in our clinical practices, we need to continue to provide services for children and adolescents with mental health disorders. CAP institutions should keep contact with patients who have special needs and those patients who are missing out on specialized education systems both to avoid disrupting current treatment programs and to offer support to caregivers. Parents and patients should be informed not to stay away from private practices and hospitals when mental health care is needed. To avoid a concentration of the most severe cases in hospitals on wards with reduced staff and difficult or unsuitable care conditions, outpatient and inpatient facilities must be kept open as much as possible; they need to follow all the necessary protocols to prevent transmission of the virus. Crisis interventions must be accessible at all times. For patients with new onset of child psychiatric disorders, appointments should be arranged and they should be directed to adequate care. Digital communication with the patient's family should be used wherever possible to support parents on how to deal with the current challenges. Europeans generally have smart phones but those in very poor families may not. They may have no opportunity for a safe, confidential space at home. Support regarding how to address anxiety and stress in children is important (for information on these issues see, e.g., recommendations of ESCAP [5]).

In many places, there are adverse consequences for training and for the workforce. Recruitment is not possible because of home working and the difficulty of ensuring equity of opportunity. This is compounded by staff being redeployed to care for COVID-19 patients. CAP is an understaffed discipline already. On the positive side, we see an acceleration in switching to online training and supervision, development of e-learning. There are newly emerging networks of international information exchange about the effects of Corona on mental health and to share lessons learned. The further development of an international network of CAP trainers as well as the development of courses on an international level and MOOCs may prove to be crucial to maintain training [6]. After the crisis, it will be important to avoid delays in training caused by the crisis. There should be regulations that acknowledge training experiences in child and adolescent psychiatry and psychotherapy even though the normal functioning of training courses, personal supervision, etc., have been interrupted. In a broader scope, in many hospitals, we are sharing what we have learned about mental health advice with other health care professionals. This may well prove to have an additional long-term effect on the awareness of the importance of (child) mental health and on recruitment for future mental health care professionals. Last but not least, mental health professionals should take precautions to safe-guard their own mental health; MindEd has recently provided front line staff in the NHS and care services with recommendations for management of mental health, whilst looking after others during the COVID-19 crisis (https://covid.minded.org.uk); child and adolescent mental health care professionals, too, may well profit from such recommendations.

Turning to the role of CAP in research, it is not an empty plea that more research is urgently needed on both the shortterm and the long-term effects on child mental health to better inform policy makers. Even though numerous reviews on mental health effects of COVID-19 are being published (just to name some examples: [7-10]), they are based on 
the existing literature that only partly mirrors the current situation. Prospective assessment of the effect of COVID19-related mental health effects in children and adolescents is needed. International collaborations in the EU seem key to both detect general as well as specific mechanisms at play in each culture and country. In addition, it is important to analyze the tele-psychiatric interventions to determine measures of quality control and ask patients, parents and therapists through questionnaires about the usefulness of online therapy to help maintain such services in the future. The question of data-protection and the use of user friendly, easy to handle systems are challenges that are not fully solved yet. However, based on the old medical ethical position that the health of the patients is the primary issue (aegroti salus suprema lex), many child and adolescent psychiatrists and psychologists and social workers have managed to stay in contact with their patients by new technical means.

Taken together, CAP faces numerous challenges. However, to maintain emergency and regular treatments, training and research wherever possible must be a priority for all CAP professionals. In the long term, child mental health is the basis for future adult mental health which is closely associated with general health and is, thus, related to productivity and well-being in our society. Although measures that aim for "social distancing" are important to protect the health of our society, political decision makers have to keep in mind that it does not come without side effects and that children, adolescents and their families bear a major burden of these measures. In medicine, treatment decisions are never made on the potential benefit alone- the risk of side effects always has to be taken into account. Likewise, in the current situation, risk-benefit analyses are urgently needed based on the effects of "social distancing" including importantly for children and adolescents school closures. Although current knowledge about the risks is based on studies with limited comparability or on just emerging novel results, this information has to be taken into account. Instead of "social distancing", measures that enable "physical distancing" with maintained "social connectedness" should be aimed. Effective alternatives for school closures may be "physical distancing" measures such as to keep students in classrooms and to decrease the number of students per class and to increase space between students $[1,11]$.

As a result of governments taking their own national measures, the return of border controls and the financial risks inherent to the COVID-19 pandemic, this crisis carries the risk of reduced solidarity across Europe. As is the case for all other international families, we, too, as the European CAP-family, find ourselves physically separated. This should not stop us from trying to remain emotionally close. Advocacy of European CAP to policy makers from a mental health perspective should be based on the principle: first, do no harm (primum non nocere).

Acknowledgements The authors of this editorial come from Europe; the contents should be interpreted accordingly. Authors include representatives/board members from the Union of European Medical Specialists, section of Child and Adolescent Psychiatry (UEMS-CAP) and the European Society of Child and Adolescent Psychiatry (ESCAP). The core author group consists of the first three authors (VC, PD and $\mathrm{JF})$.

\section{References}

1. Viner RM, Russell SJ, Croker H, Packer J, Ward J, Stansfield C, Mytton O, Bonell C, Booy R (2020) School closure and management practices during coronavirus outbreaks including COVID19: a rapid systematic review. Lancet Child Adolesc Health. https ://doi.org/10.1016/s2352-4642(20)30095-x

2. UNESCO (2020) COVID-19 educational disruption and response. https://en.unesco.org/covid19/educationresponse. Accessed 17 April 2020

3. Fegert JM, Berthold O, Clemens V, Kölch M (2020) COVID19 pandemie: kinderschutz list systemrelevant. Dtsch Arztebl Int 117(14):703

4. Armitage R, Nellums LB (2020) Considering inequalities in the school closure response to COVID-19. Lancet Glob Health. https ://doi.org/10.1016/s2214-109x(20)30116-9

5. European society for child adolescent psychiatry (2020) Dealing with children and adolescent mental health during the coronavirus pandemic. https://www.escap.eu/index/coronavirus-andmental-health/dealing-with-children-and-adoelscent-mental-healt h-during-the-coronavirus-pandemic. Accessed 17 April 2020

6. Deschamps P, Hebebrand J, Jacobs B, Robertson P, Anagnostopoulos DC, Banaschewski T, Birkle SM, Dubicka B, Falissard B, Giannopoulou I, Hoekstra PJ, Kaess M, Kapornai K, Klauser P, Revet A, Schroder CM, Seitz J, Seker A, Signorini G (2020) Training for child and adolescent psychiatry in the twenty-first century. Eur Child Adolesc Psy 29(1):3-9. https://doi.org/10.1007/s0078 7-019-01467-6

7. Brooks SK, Webster RK, Smith LE, Woodland L, Wessely S, Greenberg N, Rubin GJ (2020) The psychological impact of quarantine and how to reduce it: rapid review of the evidence. Lancet 395(10227):912-920. https://doi.org/10.1016/s0140 $-6736(20) 30460-8$

8. Torales J, O’Higgins M, Castaldelli-Maia JM, Ventriglio A (2020) The outbreak of COVID-19 coronavirus and its impact on global mental health. Int J Soc Psyc 207(64020):212-915. https://doi. org/10.1177/0020764020915212

9. Lee J (2020) Mental health effects of school closures during COVID-19. Lancet Child Adolesc Health. https://doi.org/10.1016/ s2352-4642(20)30109-7

10. Golberstein E, Wen H, Miller BF (2020) Coronavirus disease 2019 (COVID-19) and mental health for children and adolescents. JAMA Pediatr. https://doi.org/10.1001/jamapediatrics.2020.1456

11. Uscher-Pines L, Schwartz HL, Ahmed F, Zheteyeva Y, Meza E, Baker G, Uzicanin A (2018) School practices to promote social distancing in K-12 schools: review of influenza pandemic policies and practices. BMC Public Health 18(1):406. https://doi. org/10.1186/s12889-018-5302-3 


\section{Affiliations}

\section{Vera Clemens ${ }^{1,2} \cdot$ Peter Deschamps ${ }^{3,4}$. Jörg M. Fegert ${ }^{2,5}$. Dimitris Anagnostopoulos ${ }^{5,6} \cdot$ Sue Bailey ${ }^{3,7}$. Maeve Doyle ${ }^{5,8}$. Stephan Eliez ${ }^{5,9}$. Anna Sofie Hansen ${ }^{3,10}$. Johannes Hebebrand ${ }^{5,11}$. Manon Hillegers ${ }^{5,12}$. Brian Jacobs $^{3,13}$. Andreas Karwautz ${ }^{5,14}$. Eniko Kiss ${ }^{5,15} \cdot$ Konstantinos Kotsis $^{5,16}$. Hojka Gregoric Kumperscak ${ }^{3,17}$. Milica Pejovic-Milovancevic ${ }^{5,18,19}$. Anne Marie Råberg Christensen ${ }^{5,20}$. Jean-Philippe Raynaud ${ }^{5,21}$. Hannu Westerinen ${ }^{3,22}$. Piret Visnapuu-Bernadt ${ }^{3,23}$}

Peter Deschamps

P.K.H.Deschamps@umcutrecht.nl

1 Trainees and Early Career CAP platform, Frederiksberg, Denmark

2 Department of Child and Adolescent Psychiatry/Psychotherapy, University Hospital Ulm, Ulm, Germany

3 UEMS-CAP, Brussels, Belgium

4 Department of Psychiatry, Utrecht University, Utrecht, the Netherlands

5 ESCAP http://www.escap.eu

6 Department of Child and Adolescent Psychiatry, National \& Kapodistrian, University of Athens, Athens, Greece

7 Chair Centre for Mental Health, London, UK

8 Department of Child and Adolescent Psychiatry Cavan/Monaghan Mental Health Service, St Davnet's Hospital, Rooskey, Monaghan, Ireland

9 Department of Psychiatry, School of Medicine, University of Geneva, Geneva, Switzerland

10 Research Unit for Child and Adolescent Psychiatry, Aalborg University Hospital, Aalborg, Denmark

11 Department of Child and Adolescent Psychiatry, University of Duisburg-Essen, Essen, Germany
12 Department of child and adolescent psychiatry/psychology, Erasmus MC-Sophia children's hospital, Rotterdam, the Netherlands

13 Child and Adolescent Psychiatry, South London and Maudsley Hospital, London, UK

14 Department of Child and Adolescent Psychiatry, Medical University of Vienna, Vienna, Austria

15 Child and Adolescent Psychiatry Unit, Pediatric Clinic, University of Szeged, Szeged, Hungary

16 Department of Psychiatry Faculty of Medicine, School of Health Sciences, University of Ioannina, Ioannina, Greece

17 Child and Adolescent Psychiatry Unit, University Clinical Center Maribor, Department of Psychiatry, Faculty of Medicine, University of Maribor, Maribor, Slovenia

18 Faculty of Medicine, University of Belgrade, Belgrade, Serbia

19 Institute of Mental Health, Belgrade, Serbia

20 Child and Adolescent Psychiatric Center, Glostrup, Denmark

21 Centre Hospitalier Universitaire de Toulouse, Toulouse, France

22 Child psychiatry, Social and Health Services, Kymenlaakso, Finland

23 Marienthal Clinic, Tallinn, Estonia 